\title{
Identical Quantum Particles and Weak Discernibility
}

\author{
Dennis Dieks • Marijn A.M. Versteegh
}

Received: 8 May 2008 / Accepted: 22 September 2008 / Published online: 3 October 2008

(C) The Author(s) 2008. This article is published with open access at Springerlink.com

\begin{abstract}
Saunders has recently claimed that "identical quantum particles" with an anti-symmetric state (fermions) are weakly discernible objects, just like irreflexively related ordinary objects in situations with perfect symmetry (Black's spheres, for example). Weakly discernible objects have all their qualitative properties in common but nevertheless differ from each other by virtue of (a generalized version of) Leibniz's principle, since they stand in relations an entity cannot have to itself. This notion of weak discernibility has been criticized as question begging, but we defend and accept it for classical cases likes Black's spheres. We argue, however, that the quantum mechanical case is different. Here the application of the notion of weak discernibility indeed is question begging and in conflict with standard interpretational ideas. We conclude that the introduction of the conceptual resource of weak discernibility does not change the interpretational status quo in quantum mechanics.
\end{abstract}

Keywords Identical particles · Weak discernibility · Leibniz's principle · Structuralism

\section{Introduction}

Both in ordinary life and in science we are accustomed to thinking in terms of individual things, objects, which differ from each other and can bear their own distinctive names. In the literature one sometimes encounters the proposal to treat the individuality involved here as primitive, or as grounded in a not empirically accessible metaphysical principle ("haecceity" or "thisness"). This would be foreign, however, to the way concepts are typically introduced in the empirical sciences. In physics, in particular, one expects some empirical distinction to be correlated to differences in physical

D. Dieks $(\varangle) \cdot$ M.A.M. Versteegh

Institute for History and Foundations of Science, Utrecht University, P.O. Box 80.010,

3508 TA Utrecht, The Netherlands

e-mail: d.g.b.j.dieks@uu.nl 
attributes. In this paper we adopt a view on the notion of individuality that agrees with this practice. We take as our starting point that entities should differ in one or more of their qualitative features, as assigned by the relevant physical theory, in order to be treated as different individuals. This is in the spirit of Leibniz's principle of the identity of indiscernibles (PII): roughly speaking, this principle says that there can only be distinct individuals if there are qualitative differences that make it possible to distinguish between them. Classical physics is in accordance with this idea: if PII is formulated in a sufficiently general way (including the notion of weak discernibility, to be explained in Sect. 2 below), it is satisfied by the objects recognized by classical physical theory (cf. [4, Ch. 2]).

In quantum theory the notion of individuality is notoriously more problematic than in classical physics. There are reasons to doubt whether so-called identical quantum particles are objects at all; in fact, conventional wisdom in modern physics is that a field-theoretical picture is more appropriate than a particle one. It has recently been claimed by Saunders [9, 10], however, that the same sophisticated version of PII that can ground individuality in classical physics, can do the same job in quantum mechanics; that "quantum particles" with an antisymmetrical wavefunction (fermions) obey this form of PII in the same way as classical objects in symmetrical configurations. It would follow that fermions are discernible individuals in exactly the same sense as these classical objects (they are "weakly discernible").

We oppose this conclusion. As we shall argue, the situation in quantum mechanics is essentially different from that in classical physics. In a nutshell, our argument is that in the case of fermion systems the introduction of the notion of weak discernibility does not change the interpretational status quo. As we shall show, the arguments against the "objecthood" of quantum particles keep their force, and as a result the application to the "particles" of PII and the notion of weak discernibility cannot even start.

We shall focus on the impact of the notion of weak discernibility within a "standard", Copenhagen-like interpretational framework. Of course, there are other interpretations, and according to some of these identical fermions are certainly individual objects. But in these alternative interpretations the individuality of the particles is grounded in differences in their physical properties, without the need to resort to the notion of weak discernibility (think, e.g., of the Bohm interpretation, in which the particles possess different positions). We stress that it is not our purpose here to take sides with any particular interpretation of quantum mechanics: we restrict our investigation to the question of whether the introduction of weak discernibility makes a difference for the discussion of quantum individuality.

\section{Weak Discernibility}

If we want to give Leibniz's principle a fundamental role in deciding individuality in physics, we must evidently first of all come to terms with the putative counterexamples that have been proposed to it in the context of classical physics. These examples concern cases in which it seems obvious that there are more than one individuals but yet there appear to be no distinguishing characteristics, so that the individuals 
are indiscernible. One famous case, proposed by Max Black [2], is that of a universe consisting of two spheres of identical chemical composition and two miles apart (in a relational space à la Leibniz, not in Newtonian absolute space where one could resort to absolute positions in order to label the spheres). Another example is provided by a universe consisting of two hands that are each other's mirror images (Kant's enantiomorphic hands); for a mathematical example one may think of the points in the Euclidean plane. The essential feature in these cases is that the objects in question have all their properties in common: both spheres in Black's example have equal material composition and both are at two miles from a sphere; similarly, Kant's hands have the same internal geometric properties and both are mirror images of a hand; and all points in the plane have exactly the same properties. So these cases appear to demonstrate that both in ordinary life and in classical physics we employ concepts of object and individuality that are independent of the presence of distinguishing qualitative differences - in violation of PII.

As Hawley points out [6], defenders of PII can respond to such examples in a variety of ways. First, they may query whether the described situations are possible at all - this does not appear to be a promising way out in our above examples, which all are possible according to the relevant physical laws. Second, if they are willing to concede this possibility, they can dispute that these situations are best described in terms of distinct but indiscernible individuals. This can take two forms: they may either argue that if a correct analysis of discernibility is employed the objects are discernible after all, or they may claim that there were no distinct objects to start with, that there is only one undivided whole.

In the just-mentioned examples the best response - so we shall argue-is to admit that these situations are physically possible and that indeed more than one objects are present in them. But these objects are not indiscernible. In order to make clear how it is possible to justify this via PII we follow Saunders $[9,10]$, who takes his clue from Quine [8], in noting that in cases like the above examples irreflexive relations are instantiated: relations entities cannot bear to themselves. This irreflexivity is the key to proving that (a generalized version of) PII is satisfied after all: if an entity stands in a relation that it cannot have to itself, there must be at least two entities.

To see in logical detail how this works, let us formalize the argument. PII can be formulated as follows, with = denoting identity:

$$
s=t \equiv \forall P(P(s) \leftrightarrow P(t)) .
$$

The universal quantifier here ranges over all physical predicates $P$ (not haecceities!). The right-hand side of the equation stipulates that $s$ and $t$ can replace each other, salva veritate, in any $P$.

There can now be various kinds of discernibility [10]. Two objects are absolutely discernible if there is a one-place predicate that applies to only one of them; relatively discernible if there is a two-place predicate that applies to them in only one order; and weakly discernible if an irreflexive two-place predicate relates them. The latter possibility is relevant to our examples. If there is an irreflexive but symmetric twoplace predicate $P(.,$.$) that is satisfied by s$ and $t$, the definition (1) requires that if $s$ and $t$ are to be identical, we must have:

$$
\forall x(P(s, x) \leftrightarrow P(t, x)) .
$$


But this is false: in any valuation in which $P(s, t)$ is true, $P(t, t)$ cannot be satisfied by virtue of the fact that $P$ is irreflexive. It follows therefore that PII is satisfied by any two individual objects that stand in an irreflexive physical relation. What is needed to dispel the impression that PII is in trouble in these cases is to apply the principle not only to monadic predicates, but also to all $n$-ary predicates (and thus to all available physical relations).

\section{Begging the Question?}

The just-sketched proof used the notion of a valuation. A valuation results from letting terms in the language (in our case: the language of the physical theory) refer to specific elements of the intended physical domain. In order to construct such a valuation, we have to distinguish the physical things we are talking about and point to them. However, actually doing this on the basis of what we are given, namely the physical properties of the objects and the relations between them, is an impossible task in the symmetrical configurations of the examples. Indeed, because of the symmetry any property or relation that can be attributed to one object can equally be attributed to any other and we can therefore not single out any specific object. It is impossible, for example, to pin down any particular point in the Euclidean plane on the basis of the properties of the plane and its points, even if we include all relational properties, because of the rotational and translational symmetries of the plane (of course, we are speaking here of the plane tout court, without an added preferred point that can function as an origin). This impossibility may seem to endanger the above argument for the validity of PII; and therefore to jeopardize the Leibniz-style individuality of our entities after all (cf. [7]).

But this difficulty is only apparent. In order that a valuation exists, and the number of elements in the domain is a well-defined quantity, it is sufficient that a function exists that maps the domain one-to-one onto a set of labels, e.g. the set $\{1,2, \ldots, n\}$ [13, p. 457]; it is not needed that we can actually construct such a labelling. In the examples we have been considering it was given in the description of the cases (two spheres, two hands, many points) that the required mappings exist, and the only remaining question was whether these several objects satisfied PII.

This difficulty is thus easily dissolved, but its discussion nevertheless highlights an important issue: we need some assurance that the domains can be thought of as consisting of elements of which there could be more than one, in order to even start testing the validity of PII. In order to see whether we are dealing with weakly discernible individuals we must already assume that there are one or more actual relata between which the relations hold. But this possibility of "splitting up the domain" is not at all a priori evident in the context of discussions of the applicability of PII. As we have already seen, one possible stance in such discussions is to argue that there is no multiplicity at all: that there is only one undivided physical system. If this is assumed no questions about the individuation of elements of the domain by means of PII have to be answered, and a simple and parsimonious ontological picture is achieved. If there is no good reason to think of the domain as consisting of several things in the first place, this option surely recommends itself. 
Summing up, our starting point was the physically motivated desire to ground individuality in empirically accessible qualitative features, by means of PII. We argued that in some cases this can only be done by focussing on irreflexive relations between the objects. But now it appears that we must already know about the existence of individual objects, between which these relations hold, before we can even apply PII; this raises the danger of circular reasoning. Let us therefore briefly pay attention to the question of whether and how PII can be applied without begging individuality questions.

\section{Relata and Relations}

If it were indeed necessary to make sure that the domains we are discussing consist of distinct objects literally prior to any application of PII, the whole project of applying PII would be self-defeating. Why should we engage in any PII arguments if we already know that there are more than one objects? Moreover, it seems that prior to employing PII we can base our judgement that there are different objects only on nonphysical grounds, involving primitive thisness, haecceity, substance or some similar metaphysical principle. French and Krause similarly comment in their discussion of weak discernibility [4, pp. 170-171]:

Doesn't the appeal to irreflexive relations in order to ground the individuality of the objects which bear such relations involve a circularity? In other words, the worry is that in order to appeal to such relations, one has already had to individuate the particles which are so related and the numerical diversity of the particles has been presupposed by the relation which hence cannot account for it.

This issue already arises for monadic predicates; it may appear that also in this case we need individual "property bearers" before we can apply any predicate. Indeed, we need something to fill the open places in the predicate symbols.

However, we need not assume that the individuality question has been solved before we consider the individuating power of the properties. We may take a "bundleview" of objects, according to which objects are regarded as bundles of properties and are not ontologically prior to their properties. In this way we can introduce the objects together with their properties. In case that the relevant properties are relational the problem appears more serious, though (see the above quotation from French and Krause); let us therefore discuss this case in some more detail.

The form taken by the "bundle idea" in the case of relational properties is actually much discussed in present-day philosophy of science, and known as a form of structuralism. The structuralist proposal in question is that relata need not be ontologically prior to the relations they have to each other [4, p. 172]: the relata are determined by the relations, as a kind of nodes in a relational network. This does not imply, however, that the relations are prior to the relata. According to the structuralist proposal relata and relations can be completely on a par, ontologically speaking: the relata may have no other properties than specified by the relations, whereas in turn the relations can only exist if they connect relata [3]. This position fits PII very well, and offers the natural route to defuse the circularity threat. 
So in our earlier examples we need not assume that there is a division of labor between "objecthood providers" that come first, and relations that are only subsequently defined on the domain. It is possible without contradiction that the relational structure that is used in PII is at the same time our only access to Leibnizean objecthood, i.e. objecthood with a qualitative grounding. The relations in our two physical examples-being at a spatial distance from each other, being each other's mirror image-indeed give us information about the presence of objects. These relations define relata that can be displaced with respect to each other, or that can be reflected and whose orientations can be compared. Such relata are actual things, objects, that differ from each other because of their mutual distance or due to their mirror-image relation, respectively. If irreflexive relations of this type apply, there must be more than one objects as relata.

As we shall illustrate in a moment, however, the mere possibility of speaking about a domain in terms of irreflexive relations is not enough to ensure such an objecthood structure. It is sometimes possible and even usual to employ properties or relations talk in situations in which there are no different objects at all-in such cases considerations about the irreflexivity of the relations obviously cannot do anything to show that the "objects" are in fact weakly discernible. This means that even within the context of a structuralist outlook it has to be decided whether the concept of object is applicable in the first place, before arguments about weak discernibility based on irreflexivity of relations make sense.

\section{Relations without Objects}

To see the possibility of relations without actual relata, consider the example of Euros in a bank account (not coins in a piggy bank, but transferable money in a real bank account). ${ }^{1}$ Imagine a situation in which by virtue of some financial regulation the Euros in a particular account can only be transferred to different one-Euro accounts. So, in a complete money transfer an account with five Euros, say, would be emptied and five different one-Euro accounts would result. In this case the Euros in the original account stand in an irreflexive relation to each other, namely "only transferable to different accounts". But this does not make them into different objects. We could of course make an attempt to exploit the irreflexive relations for the purpose of distinguishing individual Euros, by labelling the Euros via the accounts they end up in. However, this means looking at the situation after the money transfer, which does not achieve anything for the purpose of distinguishing between the Euros in the account they are actually in, before the transfer. The essential point is that the relations here do not relate occurrent, actual features of the situation; they do not connect actual relata. Rather, the relational structure is defined with respect to what would result if the actual situation were changed. There is of course no doubt that five different oneEuro accounts (with different account numbers) are five individuals; but this does not mean that it makes sense to consider the five Euros as individual objects when they

\footnotetext{
${ }^{1}$ The example of Euros on a bank account is a standard example of non-individuals, which we think is illustrative, even though it is a bit outside physics proper.
} 
are still in one common account. On the contrary, the case of more than one money units in one bank account is the standard example to illustrate absence of individuality; it is a case in which only the account itself, with the total amount of money in it, can be treated as possessing individuality [11, 12]. Although we are accustomed to using relations and things talk here, there is nothing in the actual situation that directly corresponds to this. Speaking of several Euros in a bank account is a façon de parler. According to our best understanding of the situation, statements like "all Euros in this account have the same value-namely one Euro" are not about individual things.

Thus, we have found an important silent presupposition in the argument for PIIbased individuality in the presence of irreflexive relations. Such relations can only be trusted to be significant for the individuality issue if they are of the sort that connect actual relata.

\section{Actuality}

Whether relations occurring in scientific theories connect and determine actual relata is decided by their meaning and the role they play within the theory in question. In cases in classical physics without particular symmetries, a feature of such "actual" relations is that they can be used to distinguish and name different relata. For example, in an arbitrary configuration of classical particles the mutual distances will unambiguously characterize each individual particle. Changing the configuration so that it becomes more symmetrical will change the values of the distances, but not the actuality of the objects. In particular, situations dealt with by classical theory will not change with respect to the actuality of the objects involved when we approach a Blackean spheres-type configuration. The discernibility in asymmetrical situations thus provides us with a test for the actuality in symmetrical situations: we are justified in assuming the existence of actual entities if breaking of the symmetry is physically possible, does not involve any change in the type of properties assigned, and results in a situation with distinguishable objects (this strategy resembles the one followed by Adams, who proposes to compare Black's spheres with spheres of which one has a very slight chemical impurity [1, p. 17]). This breaking of the symmetry is like introducing a coordinates origin in describing a figure in plane geometry. If a mapping between objects and natural numbers exists in the presence of such an origin, our theoretical accounts will still be compatible with the existence of such a mapping when the reference point has been removed; what changes is merely the constructibility of the mapping. The possibility of actually naming and distinguishing disappears when we forget about the standard-however, this does not collapse the different objects into one. They still are the kind of entities that have, e.g., actual distances to other entities in the structure, even though the symmetry makes it impossible to assign names [4, p. 265].

Indeed, why do we feel so sure that there are two Blackean spheres and two Kantian hands? Our mind's eye sees Black's spheres at different distances, and Kant's hands with different orientations, before us; when we break the symmetry of the configurations in these cases by imagining ourselves or some other standard as points of 
reference, the relations with respect to this reference point make it possible to distinguish the entities. Thus we can name Black's spheres via their unequal distances to a fixed point and in Kant's universe we may imagine a reference hand conventionally called "left". Another example, relevant for our subsequent discussion of quantum objects, is furnished by two oppositely directed arrows in an otherwise empty Leibnizean world. If we fix a standard of being "up", we break the symmetry and the individual arrows become absolutely discernible as up or down.

These cases are to be contrasted with the case of the Euros in a bank account. Even though the Euros have different destinations this cannot be used to distinguish actual Euros in the account. According to our best available way of describing the situation there is a total amount of money in the account, but this amount is not composed of individual Euros.

\section{The Quantum Case}

A notorious interpretational problem of quantum theory concerns so-called identical particles. These are of the same kind, that is "they" possess the same intrinsic properties (like mass, charge, spin); e.g., electrons, protons or neutrons. It is a principle of quantum mechanics that the state of a collection of such particles is completely symmetrical (in the case of bosons) or anti-symmetrical (fermions). This symmetrization postulate implies that all one-particle states represented in the total state of a collection of identical particles occur symmetrically in it. It follows from this symmetry that any property or relation that can be attributed, on the basis of the total quantum state, to any one particle is attributable to all others as well.

The standard response within physics is to say that identical quantum particles "lack individuality". This is an awkward way of saying that there are no different particles at all: although there is traditional talk of "many of them", this should be understood in the same way as talk about many Euros in a bank account. In principle it is better, according to this received view, to renounce talk that suggests the existence of individual particles and to reconceptualize the situation in terms of the excited states of a field (analogous to thinking of the Euros in an account as one sum of money).

However, the situation is also reminiscent of the symmetric configurations of classical objects described in the previous section. As we have seen there, symmetry is not decisive for proving the absence of Leibniz-style individuality: particles may well be weakly discernible individuals. Could it not be that in the quantum case there are irreflexive physical relations between particles that guarantee their individuality in the same way as they did for Black's spheres, Kant's hands and Euclid's points? This is the position adopted by Saunders, at least for the case of fermions [10]. Indeed, the anti-symmetry of the state of many-fermions systems seems to imply the existence of irreflexive relations between components of the total system: intuitively speaking, the fermions in any pair stand in the relation of "occupying different one-particle states", even though the particles do not receive individually different quantum mechanical state descriptions. It is true (and noted by Saunders) that for bosons with their symmetrical states this manoeuvre is not available, so that collections of identical bosons are still best understood as one whole. But the conclusion that standard 
quantum mechanics entails that fermions are ordinary individuals - although only weakly discernible - is surprising and highly significant by itself.

The technical details of the argument can be illustrated by the example of two fermions in the singlet state. If $|\uparrow\rangle$ and $|\downarrow\rangle$ stand for states with spins directed upwards and downwards in a particular direction, respectively, the anti-symmetrization principle requires that a typical two-fermion state looks like

$$
\frac{1}{\sqrt{2}}\left\{|\uparrow\rangle_{1}|\downarrow\rangle_{2}-|\downarrow\rangle_{1}|\uparrow\rangle_{2}\right\},
$$

in which the subscripts 1 and 2 refer to the one-particle state-spaces of which the total state-space (a Hilbert space) is the tensor product. These two one-particle statespaces are the available candidates for the description of single particles and their labels are candidate names for the individual fermions (for the moment, we are assuming hypothetically that the notion of different individuals makes sense). Now, the anti-symmetry of the total state implies that the state restricted to state-space 1 is the same as the restricted state defined in state-space 2. (The "partial traces" are $\frac{1}{2}\{|\uparrow\rangle\langle\uparrow|+| \downarrow\rangle\langle\downarrow|\}$ in both cases.) The total spin has the definite value 0 in state (3); that is, state (3) is an eigenstate of the operator $S_{1} \otimes I+I \otimes S_{2}$. Therefore, it seems natural to say that the two spins are oppositely directed. On the other hand, we cannot assign a definite spin direction to the single particles because the up and down states occur symmetrically in each of the states in the Hilbert spaces 1 and 2, respectively.

This situation appears essentially the same as the one of the two arrows. In that case, it was not possible to designate one of the arrows as up and the other as downbut nevertheless there had to be two individual arrows in view of the oppositeness of their directions. Similarly, in the fermion case with total spin zero we apparently are dealing with two individual quantum objects with opposite spins.

\section{Quantum Individuals?}

On closer examination the similarity starts to fade away, however. One should already become wary by the observation that the irreflexive relations in the quantum case have a theoretical representation that is quite different from that of their classical counterparts. There the relations could be formalized by ordinary predicates that can be expressed as functions of occurrent properties of the individual objects (like "up" and "down" with respect to a conventionally chosen standard, or +1 and -1 ), with the correlation expressed by the fact that the sum of these two quantities has a fixed value. By contrast, in quantum theory the correlation is expressed in a more complicated way: the state of the total system is an eigenstate of a linear operator in the total system's Hilbert space. Concomitant with this formal difference is an essential difference in interpretation: according to standard interpretational ideas, ${ }^{2}$

\footnotetext{
${ }^{2}$ There are certainly also interpretations that interpret the quantum state in terms of occurrent physical properties, even if no measurements are performed. Examples are the Bohm interpretation and modal interpretations. These interpretations treat the fermions as systems with different physical properties, so that they do not need the notion of weak discernibility. Our present discussion is about whether the notion of weak discernibility makes a difference within standard interpretational ideas.
} 
quantum states should be interpreted in terms of possible measurement results and their probabilities, rather than in terms of occurrent properties. In the case at hand, a system in an eigenstate of the total spin operator with eigenvalue 0 , this means that a measurement of the total spin will with probability 1 have the outcome 0 . In this special case, in which the outcome is certain (probability 1), it is-according to the same standard interpretational ideas-harmless to assume that the total system possesses the property "total spin 0" also independently of measurement; but even so, this total spin cannot be understood as being composed of definite individual spin values of the two subsystems. Although it is of course possible to perform individual spin measurements on the subsystems (whose possible outcomes and corresponding probabilities are predictable from the total quantum state), there are no corresponding occurrent spin properties in the subsystems, independently of measurement. In the singlet state (3) the prediction of quantum mechanics is that individual spin measurements will with certainty yield opposite results, summing up to 0; but on pain of running into well-known paradoxes and no-go theorems it cannot be maintained that these results reveal oppositely directed spins that were already there before the measurements. This is an example of the notorious "holism" of quantum mechanics: definite properties of a composite system do not always supervene on definite properties of its parts.

This suggests that the correct analogue to the quantum case is not provided by two oppositely directed classical arrows, but rather by a two-Euro account that can be transformed (upon "measurement", i.e. the intervention brought about by a money transfer) into two distinct one-Euro-accounts.

To investigate further whether or not the quantum relations connect actual physical systems, we can copy the strategy followed in the classical case, namely breaking the symmetry and seeing whether in the resulting situation the quantum relations can serve as name-givers. However, this cannot work as long as we stay within a many-fermions system: quantum mechanics forbids fermion systems that are not in an anti-symmetric state-it is a matter of lawlike principle that the only relations fermions can possess with respect to each other are perfectly symmetrical. This is a significant difference from the symmetrical classical cases, in which the symmetry was contingent and the theory allowed evolutions from symmetrical to asymmetrical configurations. By contrast, in quantum mechanics the mutual relations between fermions cannot serve to distinguish individual component systems as a matter of principle. The theory does not allow any asymmetrical situations with which to approach the symmetrical situation, and our earlier test fails.

It is true that this by itself does not prove that there are no individual fermionscompare with the situation in a hypothetical world in which laws stipulate that classical spheres can only occur in symmetric configurations. In such a world we would still have good reasons to think in terms of individual spheres, e.g. because our theories could allow for an external object serving as a point of reference that makes the spheres discernible. Analogously we can try to break the symmetry in the fermion case by the introduction of a standard that is external to the fermion system itself. Quantum mechanics does not require symmetry of the total state of a fermion system plus something else, and with such an external standard in hand we may hope to be able to distinguish individual fermions. If successful, this manoeuvre would lend support to the idea that the quantum relations are of the kind to connect actual particles; 
we would have evidence that it makes sense to speak about actually existing fermions at all.

To see the inevitability of a negative outcome of any such test, consider an arbitrary system of identical quantum particles to which a gauge system has been added without any disturbance of the original system (i.e., the total state is the product of the original symmetrical or anti-symmetrical identical particles state and the state of the gauge system). Let the new total state be denoted by $|\Psi\rangle$. Any quantum relation in this state between the gauge system $g$ and one of the identical particles, described in subspace $j$, say, has the form $\langle\Psi|A(g, j)| \Psi\rangle$. Here $A(g, j)$ is a hermitian operator working in the state-spaces of the gauge system $g$ and identical particle $j$. We can now use the (anti)-symmetry of the original identical particles state to show that the gauge system stands in exactly the same relations to all "identical particles". The (anti)-symmetry entails that $P_{i j}|\Psi\rangle= \pm|\Psi\rangle$, where $P_{i j}$ stands for the operator that permutes identical particle indices $i$ and $j$. Now,

$$
\begin{aligned}
\langle\Psi|A(g, j)| \Psi\rangle & =\left\langle P_{i j} \Psi|A(g, j)| P_{i j} \Psi\right\rangle \\
& =\left\langle\Psi\left|P_{i j}^{-1} A(g, j) P_{i j}\right| \Psi\right\rangle=\langle\Psi|A(g, i)| \Psi\rangle .
\end{aligned}
$$

In other words, any quantum relation the gauge system has to $j$, it also has to $i$, for arbitrary values of $i$ and $j$. That means that these quantum relations have no discriminating value in the situation as it actually is, without measurement interventions and the changes caused by them.

It must be stressed that if the situation is changed by a measurement interaction, distinct individual results will arise, ${ }^{3}$ just as in the case of the opposite spin results of measurements in the total spin 0 state (3). But we are here interested in the question of whether a many-fermions system as it is can be regarded as a collection of weakly discernible individuals; not in the question of whether such a system can be transformed into a collection of individuals. ${ }^{4}$ With regard to the first question, the above discussion tells us that fermions are like money units in a bank account. It does not matter what external standard we introduce, it will always possess the same relations to all (hypothetically present) entities. This leaves us without a good reason to suppose that there are any actual objects composing many-fermions systems. ${ }^{5}$ This is an essential difference with the earlier examples of classical weakly discernible objects.

\section{Conclusion}

There is thus an important contrast between quantum mechanical many-fermion systems and classical collections of weakly discernible objects. In the latter case we

\footnotetext{
${ }^{3}$ The expressions $\langle\Psi|A(g, j)| \Psi\rangle$ are in this case interpreted as expectation values, averages over very many experimental trials.

${ }^{4}$ How a collection of individuals can be formed in a quantum measurement process is a question that falls outside the scope of this article, but one of the authors is hoping to address this question in a later publication.

${ }^{5}$ As Hawley [5] rightly observes, PII is in the spirit of a sober empiricism, and it fits into the programme of implementing PII to reject the introduction of component systems if there is no clear evidence in their favour.
} 
have good reasons to think that there are objects that are nameable in abstracto, although the symmetry of the situation makes it impossible to actually assign names. Application of the concept of weak discernibility shows that nevertheless there is no conflict with Leibniz's principle. The strangeness of the quantum case runs much deeper. There is no sign within the standard interpretation of quantum mechanics that "identical fermions" are things at all; there is no ground for the supposition that the quantum relations "between fermions" connect any actual physical objects. The irreflexivity of these relations does not help us here. Quantum relations have a standard interpretation not in terms of what is actual, but rather via what could happen in case of a measurement. Their irrelevance for the question of whether there are actual objects is further shown by the fact that they cannot be used in any name-giving procedure, not even after the introduction of an external standard. As far as standard quantum mechanics goes, there are no separate individual fermions and the question of whether they are weakly discernible does not even arise. Conventional wisdom, saying that systems of identical quantum particles are best considered as one whole, like an amount of money in a bank account, appears to be eminently defensible even in the face of weak discernibility.

Acknowledgements We thank Simon Saunders for comments on an earlier version.

Open Access This article is distributed under the terms of the Creative Commons Attribution Noncommercial License which permits any noncommercial use, distribution, and reproduction in any medium, provided the original author(s) and source are credited.

\section{References}

1. Adams, R.M.: Primitive thisness and primitive identity. J. Philos. 76, 5-26 (1979)

2. Black, M.: The identity of indiscernibles. Mind 61, 153-164 (1952)

3. Esfeld, M., Lam, V.: Moderate structural realism about space-time. Synthese 160, 27-46 (2008)

4. French, S., Krause, D.: Identity in Physics: A Historical, Philosophical, and Formal Analysis. Oxford University Press, Oxford (2006)

5. Hawley, K.: Weak discernibility. Analysis 66, 300-303 (2006)

6. Hawley, K.: Identity, indiscernibility and number. Unpublished manuscript (2007)

7. Keränen, J.: The identity problem for realist structuralism. Philos. Math. 9, 308-330 (2001)

8. Quine, W.V.: Grades of discriminability. J. Philos. 73, 113-116 (1976). Reprinted in Quine, Theories and Things. Harvard University Press, Cambridge (1981)

9. Saunders, S.: Physics and Leibniz's principles. In: Brading, K., Castellani, E. (eds.) Symmetries in Physics: Philosophical Reflections. Cambridge University Press (2003)

10. Saunders, S.: Are quantum particles objects? Analysis 66, 52-63 (2006)

11. Schrödinger, E.: Science and Humanism. Cambridge University Press, Cambridge (1952). Partly reprinted as: What is an elementary particle? In: Castellani, E. (ed.) Interpreting Bodies: Classical and Quantum Objects in Modern Physics, pp. 197-210. Princeton University Press, Princeton (1998)

12. Teller, P.: Quantum mechanics and haecceities. In: Castellani, E. (ed.) Interpreting Bodies: Classical and Quantum Objects in Modern Physics, pp. 114-141. Princeton University Press, Princeton (1998)

13. Van Fraassen, B.C.: Quantum Mechanics-An Empiricist View. Clarendon Press, Oxford (1991) 